\title{
Search for nanosecond-fast optical transients with
}

\section{TAIGA-HiSCORE array}

A. D. Panov, ${ }^{a, *}$ I. I. Astapov, ${ }^{b}$ A. K. Awad, ${ }^{e}$ G. M. Beskin, ${ }^{c}$ P. A. Bezyazeekov, ${ }^{d}$ M. Blank, ${ }^{e}$ E. A. Bonvech, ${ }^{a}$ A. N. Borodin, ${ }^{f}$ M. Brückner, ${ }^{g}$ N. M. Budnev, ${ }^{d}$ A. V. Bulan, ${ }^{a}$ D. V. Chernov, ${ }^{a}$ A. Chiavassa, ${ }^{h}$ A. N. Dyachok, ${ }^{d}$ A. R. Gafarov, ${ }^{d}$ A. Yu. Garmash, ${ }^{i, j}$ V. M. Grebenyuk, ${ }^{f}$ O. A. Gress, ${ }^{d}$ T. I. Gress, ${ }^{d}$ A. A. Grinyuk, ${ }^{f}$ O. G. Grishin, ${ }^{d}$ D. Horns, ${ }^{e}$ A. L. Ivanova, ${ }^{j, d}$ N. N. Kalmykov, ${ }^{a}$ V. V. Kindin, ${ }^{d}$ S. N. Kiryuhin, ${ }^{d}$ R. P. Kokoulin, ${ }^{b}$ K. G. Kompaniets, ${ }^{d}$ E. E. Korosteleva, ${ }^{a}$ V. A. Kozhin, ${ }^{a}$ E. A. Kravchenko, ${ }^{i, j}$ A. A. Krivopalova, ${ }^{r}$ L. A. Kuzmichev, ${ }^{a}$ A. P. $K r y u k o v,{ }^{a}$ A. A. Lagutin, ${ }^{k}$ M. V. Lavrova, ${ }^{f}$ Yu. Lemeshev, ${ }^{d}$ B. K. Lubsandorzhiev, ${ }^{l}$ N. B. Lubsandorzhiev, ${ }^{a}$ A. D. Lukanov, ${ }^{l}$ D. Lukyantsev, ${ }^{d}$ R. R. Mirgazov, ${ }^{d}$ R. Mirzoyan, ${ }^{m, a}$ R. D. Monkhoev, ${ }^{d}$ E. A. Osipova, ${ }^{a}$ A. L. Pakhorukov, ${ }^{d}$ L. A. Panasenko, ${ }^{i}$ A. Pan,${ }^{f}$ L. V. Pankov, ${ }^{d}$ A. A. Petrukhin, ${ }^{b}$ D. A. Podgrudkov, ${ }^{a}$ V. A. Poleschuk, ${ }^{d}$ M. Popesku, ${ }^{n}$ E. G. Popova, ${ }^{a}$ A. Porelli, ${ }^{g}$ E. B. Postnikov, ${ }^{a}$ V. V. Prosin, ${ }^{a}$ V. S. Ptuskin, ${ }^{o}$ A. A. Pushnin, ${ }^{d}$ R. I. Raikin, ${ }^{k}$ A. Razumov, ${ }^{a}$ E. Rjabov, ${ }^{d}$ G. I. Rubtsov, ${ }^{l}$ Y. I. Sagan, ${ }^{f, p}$ V. S. Samoliga, ${ }^{d}$ A. Yu. Sidorenkov, ${ }^{l}$ A. A. Silaev, ${ }^{a}$ A. A. Silaev (junior), ${ }^{a}$ A. V. Skurikhin, ${ }^{a}$ M. Slunecka, ${ }^{f}$ A. V. Sokolov, ${ }^{i, j}$ Y. Suvorkin, ${ }^{d}$ L. G. Sveshikova, ${ }^{a}$ V. A. Tabolenko, ${ }^{d}$ A. B. Tanaev, ${ }^{d}$ B. A. Tarashansky, ${ }^{d}$ M. Ternovoy, ${ }^{d}$ L. G. Tkachev,,${ }^{f, p}$ M. Tluczykont, ${ }^{e}$ N. Ushakov, ${ }^{l}$ A. Vaidyanathan, ${ }^{i}$ P. A. Volchugov, ${ }^{a}$ N. V. Volkov, ${ }^{k}$ D. Voronin, ${ }^{l}$ R. Wischnewski, ${ }^{g}$ I. I. Yashin, ${ }^{b}$ A. V. Zagorodnikov $^{d}$ and D. P. Zhurov ${ }^{d}$

\footnotetext{
${ }^{a}$ Lomonosov Moscow State University Skobeltsyn Institute of Nuclear Physics (MSU SINP), Leninskie gory 1(2), GSP-1, Moscow, 119991, Russia

${ }^{b}$ National Research Nuclear University MEPhI (Moscow Engineering Physics Institute), Kashirskoe highway 31, Moscow, 115409, Russia

${ }^{c}$ Special Astrophysical Observatory, Nizhnij Arkhyz, Zelenchukskiy region, Karachai-Cherkessian Republic, 369167, Russia

${ }^{d}$ Institute of Applied Physics, Irkutsk State University (API ISU), Gagarin Blvd. 20, Irkutsk, 664003, Russia

${ }^{e}$ Institute of experimental physics of Hamburg University, Luruper Chaussee 149, 22761 Hamburg, Germany

${ }^{f}$ Joint Institute for Nuclear Research, Joliot-Curie 6, Dubna, Moscow Region, 141980, Russia

${ }^{g}$ Deutsches Elektronen-Synchrotron DESY, 15738 Zeuthen, Germany

${ }^{h}$ Physics Department of the University of Torino and the National Institute of Nuclear Physics INFN, 10125 Torino, Italy

${ }^{i}$ Novosibirsk State University, Pirogova 1, Novosibirsk, 630090, Russia

${ }^{j}$ Budker Institute of Nuclear Physics of the Siberian Branch of the Russian Academy of Sciences, Lavrentyev Prosp. 11, Novosibirsk, 630090, Russia
}

${ }^{*}$ Presenter 
${ }^{k}$ Altai State University, Lenina 61, Barnaul, 656049, Russia

${ }^{l}$ Institute for Nuclear Research of the Russian Academy of Sciences, 60th October Anniversary 7a, 117312, Moscow, Russia

${ }^{m}$ Max Planck Institute for Physics, Fohringer Ring 6, D-80805 Munich, Germany

${ }^{n}$ Institute of Space Science, P.O.Box MG-23, Ro 077125, Bucharest-Magurele, Romania

${ }^{o}$ Pushkov Institute of Terrestrial Magnetism, Ionosphere and Radio Wave Propagation of the Siberian Branch of the Russian Academy of Sciences (IZMIRAN), Kaluzhskoe highway 4, Moscow, Troitsk, 108840, Russia

${ }^{p}$ Dubna State University, Universitetskaya 19, Dubna, Moscow region, 141982, Russia

${ }^{r}$ Science-Cultural Center SETI, Sternberg Astronomical Institute, Moscow State University, Universitetsky pr., 13, Moscow 119234, Russia

E-mail: panov@dec1.sinp.msu.ru, kuz@dec1.sinp.msu.ru

The wide-angle Cherenkov array TAIGA-HiSCORE (FOV $\sim 0.6$ ster), was originally buit as part of the TAIGA installation for high-energy gamma-ray astronomy and cosmic ray physics. It was proven that the array can also be used to search for nanosecond events in the optical range. This report discusses the method of searching for optical transients using the HiSCORE array and demonstrates its performance on a real example of detecting signals from a man-made Earth satellite. Search for short flares in the HiSCORE data of winter season 2018-2019 is carried out. One candidate for double optical repeater has been detected, but the estimated probability of a random chance of such a transient by background EAS is 78\%. An upper limit on the probability of optical spikes with flux density of more than $10^{-4} \mathrm{erg} / \mathrm{sec} / \mathrm{cm}^{2}$ and a duration more than $5 \mathrm{~ns}$ is established as $\sim 2 \times 10^{-3}$ events/sr/hour.

$37^{\text {th }}$ International Cosmic Ray Conference (ICRC 2021)

July 12th - 23rd, 2021

Online - Berlin, Germany 


\section{Introduction}

The astrophysical complex TAIGA (Tunka Advanced Instrument for cosmic ray physics and Gamma-ray Astronomy) [1-4] is located in the Tunka valley, at about $50 \mathrm{~km}$ to the West, near the Baikal Lake. It is designed for the study of cosmic rays and gamma-rays of high and ultrahigh energies using the technique of observation of extensive air showers (EAS). The complex includes several instruments, including the TAIGA-HiSCORE wide-aperture Cherenkov array. The HiSCORE array (High Sensitivity COsmic Rays and gamma Explorer) consists of integrated optical atmospheric Cherenkov stations, which include four PMTs, with a total entrance of about $0.5 \mathrm{~m}^{2}$ and a field of view (FoV) of 0.6 steradian. All stations are oriented in the same way (at present they are align for observing the Crab Nebula). The stations form an array with a step of $106 \mathrm{~m}$. The HiSCORE array is still in the process of construction, and it currently consists of 120 stations located on an area of more than $1 \mathrm{~km}^{2}$. The design and construction details of the HiSCORE array are presented in the articles $[2,5]$. The operating principle of the TAIGA-HiSCORE array is based on the idea presented in the article [5]: the detector stations measure the amplitudes of Cherenkov light from EAS, as well as the arrival times to reconstruct the direction of arrival of a cosmic ray particle (charged or gamma quantum), its initial energy, and some other important characteristics of EAS from these data.

The characteristic duration of EAS Cherenkov light pulse ranges from several nanoseconds to tens of nanoseconds; the HiSCORE detector stations are adapted to register just such flashes. The detection threshold is approximately 3000 green photons $(\sim 530 \mathrm{~nm})$ per square meter during an integration time of $10 \mathrm{~ns}$. The array is capable of detecting not only EAS Cherenkov light pulses, but any flashes of light. In this case, according to a number of characteristics, a splash of a distant point source (hereinafter referred to as optical transient) in the most cases can be easily distinguished from a passage of a Cherenkov light front of EAS. Indeed, the stations triggered by an EAS Cherenkov light pulse will cluster, as a rule, around the shower axis. On the contrary, distant transient events generate a flat and homogeneous optical front that evenly covers the entire HiSCORE array. This idea is confirmed by comparing the structure of a typical EAS event and an event caused by a pulse of a laser installed on the orbital satellite (Fig. 1). The signal from the satellite flying over the HiSCORE array was unexpectedly detected in the data for December 10, 2018 (see the trajectory of the sattelite in Fig. 2) and was subsequently identified as the signal from the LIDAR on the CALIPSO satellite [6]. Further, it was found that the signal from CALIPSO has been present in the data since 2015 and can be used for the absolute pointing calibration of HiSCORE array [6] just as it was obtained using signals from the LIDAR on ISS [7]. Thus, the HiSCORE Cherenkov array, originally adapted for the study of cosmic ray physics and for gamma-ray astronomy, can be relatively easily adopted for solving some problems of conventional optical astronomy. An interesting question is what kind of distant astrophysical sources can lead to nanosecond flashes of light that could be detected by HiSCORE. If the source is not coherent, then the size of the emitter should be measured at most in units of meters, since in $1 \mathrm{~ns}$ light passes travels $0.3 \mathrm{~m}$ in air and vacuum. If the source of the pulse is coherent, then there are no restrictions on the size of the source, but in this case we should talk about a quantum generator of either natural or extraterrestrial origin. Natural cosmic sources of coherent radiation, including optical lasers, are known [8], but such sources emit in a continuous mode. The most understandable hypothetical 

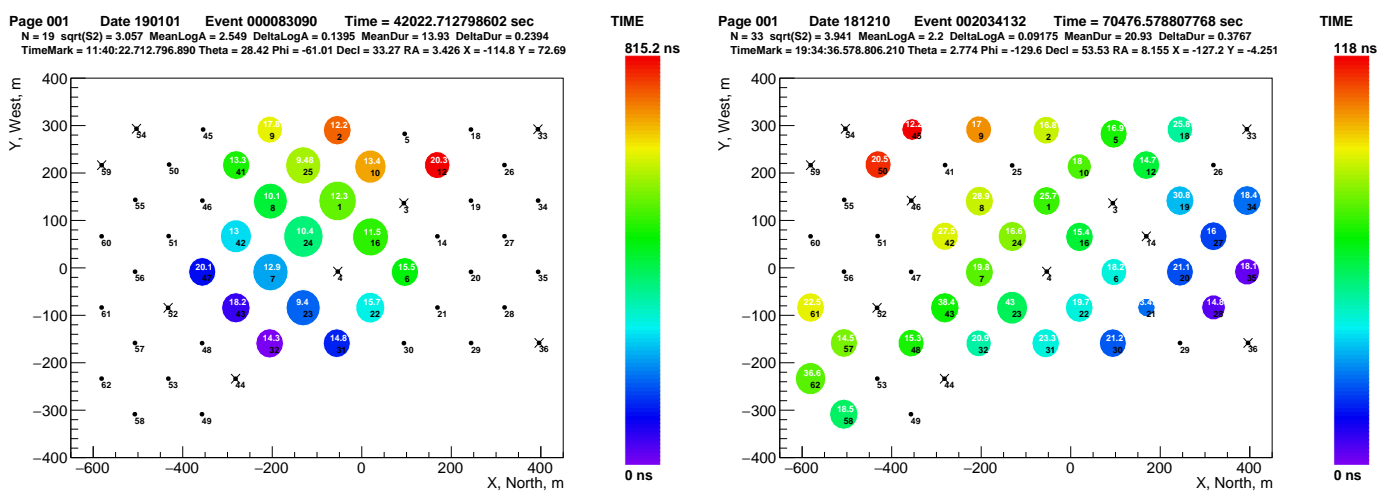

Figure 1: Portraits of a typical EAS event (left diagram) and an event of a distant optical transient, which is represented by a laser pulse (LIDAR) from a distance of about $700 \mathrm{~km}$. The diagrams show the response times of the optical stations in color, and the estimates of the signal duration in nanoseconds are inscribed in the colored circles of the triggered stations in numbers. The size of the circles represents the amplitude of the signal in a logarithmic scale. The title of each portrait contains a variety of technical information about the event. The numbers of the stations of the HiSCORE telescope are also shown, while the crosses indicate the stations that at the time of measuring of the event for some reason were not functioning or had operational problems. The configuration of the HiSCORE array corresponds to the winter measurement season of 2018-2019.

source of short optical pulses is a distant optical laser of artificial origin, that is, an alien laser. Thus, the task is within the framework of the SETI problem (Search for Extra Terrestrial Intelligence). It is easy to understand that even for very large distances, a laser with relatively modest parameters is sufficient for its radiation to be detected by HiSCORE optical stations. For example, for detecting a signal from the hypotetical transmitter with an aperture of $1000 \mathrm{~m}$, for light of $530 \mathrm{~nm}$ (green), at a distance of $10^{4}$ ly from the Solar System, the laser energy should be about $9 \mathrm{MJ}$ for every $10 \mathrm{~ns}$ pulse. An aperture of $1000 \mathrm{~m}$ means the use of a laser phased array.

Note that the idea of using large Cherenkov telescopes to search for optical transients of astrophysical origin was proposed already at the very beginning of the 2000s [9], including also the framework of the SETI problem [10]. After that, the idea was repeatedly discussed in various aspects and some search programs were implemented [11-15]. However, in all these works, it was assumed and discussed the use of image Cherenkov telescopes, which have a small field of view of only several degrees. The HiSCORE Cherenkov array has a very wide field of view, which covers about one tenth of the visible sky. This is really important exactly in the SETI searches when it comes to detecting very rare events that may appear from an unpredictable direction and time.

\section{Methods}

In this work, we study the data of the HiSCORE array recorded in the winter season from November 2018 to April 2019, when the TAIGA-HiSCORE array included 54 optical stations. The observations were made during 80 clear, moonless nights with a total observation time of 475 hours and an exposure of $288 \mathrm{sr} \cdot \mathrm{hr}$. 
If trigger of optical stations is caused by a distant optical transient, then the maximum pulse amplitudes of all stations should ideally be the same up to statistical fluctuations. For this reason, one of the important criteria in the search for candidates for optical transients is the comparison of the amplitudes of the stations. However, the amplification factor of different stations are not quite the same; therefore, a special relative amplitude calibration is required, which makes it possible to compare the amplitudes of different stations of the same event. The idea behind this calibration is very simple. During each night of measurements, all optical stations are in the same conditions, having on average the same flux of EAS optical pulses at the input. Therefore, equally operating stations should show the same amplitude spectra. In reality, this is not the case due to differences in the amplification factors of the stations. The idea of the calibration that has been performed was to select such a correction factor for the amplitudes for each optical station so that all spectra of the stations are reduced to some single average spectrum, the same for all stations.

The most important task of HiSCORE data processing is the reconstruction of the direction to the source, which is carried out using the measured response times of different optical stations caused by the passage of the light front. In this work, the direction is reconstructed under the assumption of a plane light front, which is correct for a distant optical transient, but also gives the direction of the EAS axis, despite the fact that the shape of the front of EAS Cherenkov light is not really flat. This is possible due to the fact that, although the EAS front is not flat, it is axially symmetric with respect to the shower axis. The problem of finding the direction to the source is reduced to minimization along two angular coordinates $-\varphi$ (azimuth) and $\theta$ (polar angle) - of some $\chi^{2}$-like function.

Several passes the CALIPSO satellite equipped with a LIDAR for observing the state of the atmosphere have been recorded by HiSCORE. The satellite's orbital altitude is $700 \mathrm{~km}$, the laser pulse duration is about $20 \mathrm{~ns}$, thus CALIPSO provides an almost ideal test for signals from a distant optical source. The scatter of the trajectory points was used to determine the accuracy of the reconstruction of the angular coordinates for events near the zenith (where the satellite appears in the HiSCORE aperture), which was about $0.05^{\circ}$.

\section{Results}

Since the event of a distant optical transient covers the entire area of the HiSCORE array uniformly, in contrast to the great majority of EAS events, it should be expected that a certain parameter representing the degree of uniformity of illumination can be effectively used to select candidates for distant transients. As such a parameter, we introduce a parameter called "event size", EventSize. The event size is understood as the maximum distance in the $(x, y)$ plane in meters between the triggered stations belonging to this event. If this criterion is used in conjunction with the condition DeltaLogA $<0.1$, where DeltaLogA is the standard deviation for the logarithm of the amplitude of triggered stations for one event, then it turns out to be very effective for selecting potential candidates for distant transients. The limitation on the spread of amplitudes for optical transient events DeltaLogA $<0.1$ was deduced from the above mentioned satellite test events (see the Methods section above). It was shown that the majority of satellite events are larger than $900 \mathrm{~m}$, while the majority of all other events are less than $900 \mathrm{~m}$, with a maximum near $600 \mathrm{~m}$. Therefore, 
if we consider events with a size greater than $900 \mathrm{~m}$, then we will get rid of most of the EAS background, preserving most of the satellite events (i.e., most of the events of distant transients).

Fig. 2 shows the distribution of all events with number of triggered stations $N_{E f f} \geq 20$, EventSize $>900 \mathrm{~m}$, DeltaLogA $<0.1$ over the sky in local and equatorial coordinates. In the region of small zenith angles, $\theta<40^{\circ}$ (or large declination angles, Decl $>25^{\circ}$ ), a track of satellite events is visible, the majority of which pass this filtering, being distant optical transients, but there are no other events. This means that there are no other candidates for distant optical transients in the region of these angles. This is one of the results of this work. The second conclusion from Fig. 2 is that virtually any "flat" event detected near zenith should be considered to be a distant optical transient candidate.

However, it can be seen from the same Fig. 2 that in the area of large zenith angles, which means the area of low declinations (including negative declinations), there is a large group of events that pass all conditions filtration for "flatness". These are rare very gentle showers that can cover the entire area of the HiSCORE array, simultaneously creating close amplitudes of different triggered optical stations, completely simulating the passage of a uniform flat front. A total of 511 such events were filtered. It is obvious that these EAS events are the background for potential events of distant optical transients. The question is, whether is it possible to find candidate events for optical transients in such a background, separating them in some way from the background events?

The answer to this question is "yes" if one looks not for single candidates for transients, but for repeater candidates, where a repeater is understood as a group of events that came from one point in the sky. Finding a repeater among the filtered 511 candidates would mean finding a real candidate for a repeating optical transient if it is shown that the random simulation of such a repeater by the EAS background is small.

Obviously, for events that come from one point in the sky the difference in angular coordinates should be less than the errors in measuring their directions. As mentioned above, an error in determining the directions of $0.05^{\circ}$ was measured for events caused by a laser mounted on the satellite. However, this result refers to events close to the zenith of the local coordinate system, while all 511 candidates are events with large zenith angles. Such events have a completely different space-time structure, and the error in determining the coordinates for them will, generally, be different. Errors of angle coordinates for such events was calculated by the Monte Carlo method according to the known distribution laws of errors in the trigger times of optical stations. As a result, the number $\Delta \varphi=0.03^{\circ}$ was obtained, which has the meaning of the standard deviation in any of the angular coordinates. It can be seen that the error obtained is indeed different from the error for events near the zenith. For the final calculations, we postulated that a group of events with the same coordinates are those for which all events are included in a circle with a center coinciding with the center of mass of all events in the group and a radius of $R=\Delta \varphi \sqrt{2}=0.041^{\circ}$.

The search among 511 candidates resulted in the discovery of one pair of events with formally indistinguishable coordinates. Calculations by the Monte Carlo method showed that the probability of chance simulation of at least one double repeater is $78 \%$. That is, the appearance of one double repeater should be expected simply by chance, therefore we cannot consider the found repeater as a real candidate for optical transients.

Thus, the main result of this work is that neither single candidates for optical transients were detected under conditions of a low background near the zenith, nor candidate repeaters with large 

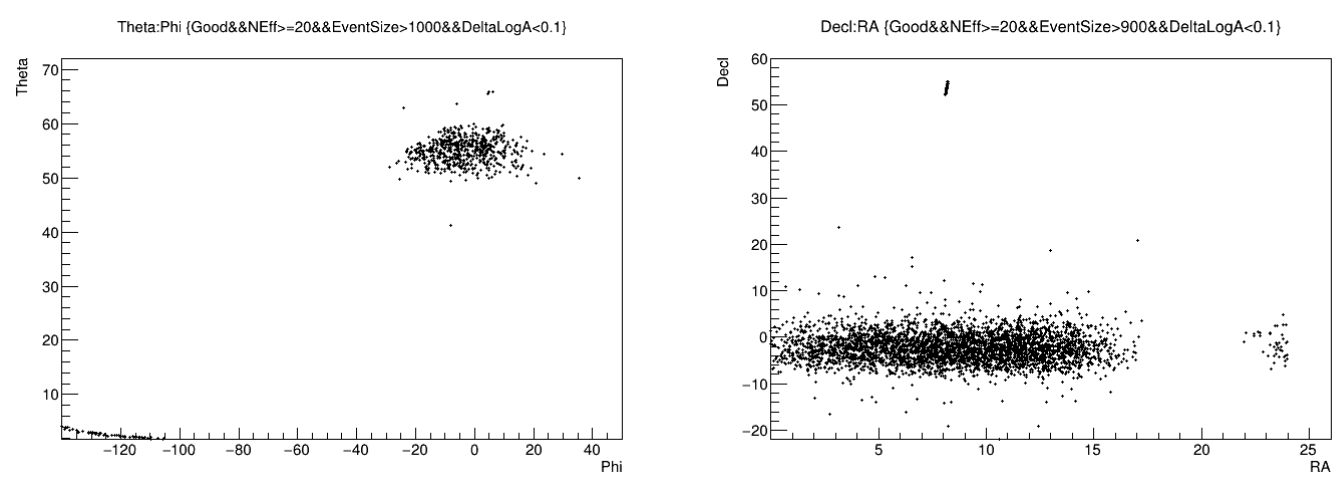

Figure 2: Distribution of events over the sky for the events with a size of more than $900 \mathrm{~m}$ and the condition DeltaLogA $<0.1$ : in the region of small zenith angles (or large declination angles) there are no events other than satellite events.

zenith angles in the background of an EAS were found. The absence of candidates for optical transients allows us to set limits on the frequency of occurrence of such events. Since the exposure for the season 2018-2019 is known (288 ster-hour), and no candidate was detected, then, taking into account the thresholds and signal duration that could be observed, the limitation on the flux of the sought transients can be formulated in the following way: for optical transients with an energy flux more than $10^{-4} \mathrm{erg} / \mathrm{cm}^{2} / \mathrm{sec}$ and pulse duration more than $5 \mathrm{~ns}$ the flow is less than $2 \times 10^{-3}$ events/ster/hour.

In conclusion, we note that the data of the HiSCORE array for the seasons 2019-2020 and 2020-2021 will be processed soon. Since the size of the array grows up every year, a noticeable improvement in the background conditions for the search for optical transients in the HiSCORE data is expected.

The work was performed at the UNU "Astrophysical Complex of MSU-ISU" (agreement 13.UNU.21.0007). The work is supported by the Russian Science Foundation (grant 19-72-20067 (Section 3), the Russian Federation Ministry of Science and High Education (projects FZZE-20200017, FZZE-2020-0024). The work was (partially) performed as part of the government contract of the SAO RAS approved by the Ministry of Science and Higher Education of the Russian Federation. G. Beskin acknowledges the support of Ministry of Science and Higher Education of the Russian Federation under the grant 075-15-2020-780 (N13.1902.21.0039).

\section{References}

[1] N. Budnev, I. Astapov, N. Barbashina, A. Barnyakov, P. Bezyazeekov, A. Bogdanov et al., The TAIGA experiment: From cosmic-ray to gamma-ray astronomy in the Tunka valley, Nuclear Inst. and Methods in Physics Research, A 845 (2017) 330.

[2] O. Gress, I. Astapov, N. Budnev, P. Bezyazeekov, A. Bogdanov, V. Boreyko et al., The wide-aperture gamma-ray telescope TAIGA-HiSCORE in the Tunka Valley: Design, composition and commissioning, Nuclear Inst. and Methods in Physics Research, A 845 (2017) 367. 
[3] L. Kuzmichev, I. Astapov, P. Bezyazeekov, A. Borodin, M. Brückner, N. Budnev et al., Cherenkov EAS arrays in the Tunka astrophysical center: From Tunka-133 to the TAIGA gamma and cosmic ray hybrid detector, Nuclear Inst. and Methods in Physics Research, A 952 (2020) 161830.

[4] N. Budnev, I.I. Astapov, P.A. Bezyazeekov, A. Borodin, M. Brückner, D. Chernykh et al., TAIGA - A hybrid array for high-energy gamma astronomy and cosmic-ray physics, Nuclear Inst. and Methods in Physics Research, A 958 (2020) 162113.

[5] M. Tluczykont, D. Hampf, D. Horns, T. Kneiske, R. Eichler, R. Nachtigall et al., The ground-based large-area wide-angle gamma-ray and cosmic-ray experiment HiSCORE, Adv. Space Res. 48 (2011) 1935.

[6] A. Porelli for the TAIGA collaboration, PoS ICRC2021 (2021) 876.

[7] A.Porelli, R.Wischnewski, A.Garmash, I. Astapov, P. Bezyazeekov, V. Boreyko et al., TAIGA-HiSCORE detection of the CATS-LIDAR on the ISS as fast moving point source, PoS ICRC2017 (2017) 754.

[8] S. Johansson and V.S. Letokhov, Astrophysical lasers and nonlinear optical effects in space, New Astronomy Reviews 51 (2007) 443.

[9] G. Beskin, C. Bartolini, A. Guarnieri, A. Piccioni, S. Biryukov, D. Eichler et al., Simultaneous detection of the high energy and optical transients by Cerenkov telescopes, in ESO Symposia: Gamma-Ray Bursts in Afterglow, pp. 387-389, Springer-Verlag, 2001.

[10] D. Eichler and G. Beskin, Optical search for extraterrestrial intelligence with air Cerenkov telescopes, ASTROBIOLOGY 1 (2001) 489.

[11] J. Holder, P. Ashworth, S. LeBohec, H.J. Rose and T.C. Weekes, Optical SETI with imahing Cherenkov telescopes, in 29th International Cosmic Ray Conference, vol. 5, (Pune), pp. 387-390, 2005.

[12] S.C. Griffin, Searching for fast optical transients using a Veritas Cherenkov telescope, in New Horizons in Time-Domain Astronomy, R. Griffin, R. Hanisch and R. Seaman, eds., no. 285 in Proceedings IAU Symposium, pp. 321-323, International Astronomical Union, 2011, DOI.

[13] I. Bartos, P. Veres, D. Nieto, V. Connaughton, B. Humensky, K. Hurley et al., Cherenkov Telescope Array is well suited to follow up gravitational-wave transients, MNRAS 443 (2014) 738.

[14] I. Bartos, T.D. Girolamo, J.R. Gair, M. Hendry, I.S. Heng, T.B. Humensky et al., Strategies for the follow-up of gravitational wave transients with the Cherenkov Telescope Array, MNRAS 477 (2018) 639.

[15] V.A. Acciari, Ansoldi, L.A. Antonelli and et. al (MAGIC Collaboration), Constraining very-high-energy and optical emission from FRB 121102 with the MAGIC telescopes, MNRAS 481 (2018) 2479. 